\title{
Brown-Vialetto-Van Laere Syndrome 1
}

National Cancer Institute

\section{Source}

National Cancer Institute. Brown-Vialetto-Van Laere Syndrome 1. NCI Thesaurus. Code C133724.

An autosomal recessive neurologic condition caused by mutation(s) in the SLC52A3 gene, encoding solute carrier family 52, riboflavin transporter, member 3 . It is characterized by sensorineural hearing loss and varying cranial nerve palsies, usually affecting the motor components of the seventh and ninth to twelfth cranial nerves. Spinal motor nerves are often affected. Mutations in SLC52A3 may result in Faxio-Lone disease, which is a similar condition, but sensorineural deafness is not present. 